OPEN ACCESS

Edited by:

Hu Zhang,

Sichuan University, China

Reviewed by:

Hakan Akin,

Marmara University, Turkey

Roberto Gramignoli,

Karolinska Institutet (KI), Sweden

*Correspondence:

Gabriella Pár

pargabriella@gmail.com

tThese authors have contributed equally to this work

Specialty section:

This article was submitted to

Gastroenterology,

a section of the journal

Frontiers in Medicine

Received: 05 November 2020 Accepted: 22 February 2021 Published: 12 March 2021

Citation:

Hegyi PJ, Váncsa S, Ocskay K Dembrovszky F, Kiss S, Farkas N, Erőss B, Szakács Z, Hegyi P and

Pár G (2021) Metabolic Associated Fatty Liver Disease Is Associated With an Increased Risk of Severe COVID-19: A Systematic Review With Meta-Analysis. Front. Med. 8:626425. doi: 10.3389/fmed.2021.626425

\section{Metabolic Associated Fatty Liver Disease Is Associated With an Increased Risk of Severe COVID-19: A Systematic Review With Meta-Analysis}

\author{
Péter Jenö Hegyi ${ }^{1 \dagger}$, Szilárd Váncsa ${ }^{1,2 \dagger}$, Klementina Ocskay ${ }^{1,2}$, Fanni Dembrovszky ${ }^{1,2}$, \\ Szabolcs Kiss ${ }^{1,2,3}$, Nelli Farkas ${ }^{1,4}$, Bálint Eröss ${ }^{1}$, Zsolt Szakács ${ }^{1,2}$, Péter Hegyi ${ }^{1,2}$ and \\ Gabriella Pár ${ }^{1,5 *}$
}

${ }^{1}$ Institute for Translational Medicine, Medical School, University of Pécs, Pécs, Hungary, ${ }^{2}$ Szentágothai Research Center, University of Pécs, Pécs, Hungary, ${ }^{3}$ Doctoral School of Clinical Medicine, University of Szeged, Szeged, Hungary, ${ }^{4}$ Institute of Bioanalysis, Medical School, University of Pécs, Pécs, Hungary, ${ }^{5}$ Division of Gastroenterology, First Department of Medicine, Medical School, University of Pécs, Pécs, Hungary

Background: The most common pre-existing liver disease, the metabolic dysfunction-associated fatty liver disease (MAFLD) formerly named as non-alcoholic fatty liver disease (NAFLD), may have a negative impact on the severity of COVID-19. This meta-analysis aimed to evaluate if MAFLD or NAFLD are associated with a more severe disease course of COVID-19.

Methods: A systematic search was performed in five databases for studies comparing severity, the rate of intensive care unit (ICU) admission, and mortality of COVID-19 patients with and without MAFLD or NAFLD. In meta-analysis, pooled odds ratios (ORs) with 95\% confidence intervals (Cls) were calculated.

Results: Altogether, we included nine studies in our quantitative and qualitative synthesis. MAFLD was associated with an increased risk of severe COVID-19 compared to the non-MAFLD group (28 vs. $13 \%$, respectively; $\mathrm{OR}=2.61, \mathrm{Cl}: 1.75-3.91)$. Similarly, in the NAFLD vs. non-NAFLD comparison, NAFLD proved to be a risk factor as well (36 vs. $12 \%$, respectively; $O R=5.22, \mathrm{Cl}: 1.94-14.03)$. On the other hand, NAFLD was not associated with an increased risk of ICU admission (24 vs. $7 \%$, respectively; OR $=2.29$, Cl: 0.79-6.63). We were unable to perform meta-analysis to investigate the association of MAFLD with the rate of ICU admission and with mortality.

Conclusion: In conclusion, patients with MAFLD and NAFLD showed a more severe clinical picture in COVID-19. Our results support the importance of close monitoring of COVID-19 patients with MAFLD. Further research is needed to explore the cause of increased severity of COVID-19 in MAFLD.

Keywords: SARS-CoV-2, COVID-19, pandemic, prognosis, non-alcoholic fatty liver disease, metabolic associated fatty liver disease 


\section{INTRODUCTION}

Severe acute respiratory syndrome coronavirus 2 (SARS-CoV-2) represents a global health challenge. Coronavirus disease 2019 (COVID-19) is mostly a self-limiting disease; however, in some cases, mortality can reach 3-7\% (1). The high mortality has been mainly linked to the excessive production of pro-inflammatory cytokines that lead to organ failure, most importantly, acute respiratory distress syndrome (ARDS) (2).

Advanced age and comorbidities, such as hypertension, chronic obstructive pulmonary disease, or cardiovascular diseases are proved risk factors in COVID-19 $(1,3)$. Patients with elements of metabolic syndrome (MS), such as diabetes, obesity, or hyperlipidemia are more susceptible to infection and also have worse outcomes in COVID-19 $(4,5)$. MS was found to be associated with chronic low-grade inflammation that compromises the immune system and causes microvascular endothelial dysfunction, which may contribute to poor outcomes in COVID-19 $(6,7)$.

Metabolic-associated fatty liver disease (MAFLD) and nonalcoholic fatty liver disease (NAFLD) are the most common chronic liver diseases (CLD), which affect about a quarter of the world's adult population (8). Pre-existing liver diseases such as NAFLD or the recently defined MAFLD, as the hepatic manifestations of MS (8), might also be significant risk factors of hospitalization and severity in COVID-19 $(9,10)$. The MAFLD criteria are based on evidence of hepatic steatosis in addition to one of the following three criteria: overweight/obesity, presence of type 2 diabetes mellitus, and proof of metabolic dysregulation (8).

According to recent publications, the presence of MAFLD and NAFLD may exacerbate the virus-induced inflammatory "storm" possibly through the hepatic release of pro-inflammatory cytokines and by increased reactive oxygen production in COVID-19 patients (11-13).

There are still limited reports on how MAFLD and NAFLD influence clinical outcomes in patients with COVID-19, and there are no meta-analytical reports of the available evidence. This meta-analysis aimed to evaluate if MAFLD or NAFLD are associated with a more severe disease course of COVID-19.

\section{METHODS AND MATERIALS}

We report our systematic review and meta-analysis following the Preferred Reporting Items for Systematic Reviews and MetaAnalyses (PRISMA) 2009 Statement (Supplementary Table 1) (14). We registered the protocol of this study onto the International Prospective Register of Systematic Reviews (CRD42020210923) and adhered to it during the course, except for including mortality in our outcomes (see https://www.crd. york.ac.uk/prospero).

\section{Search and Selection}

A systematic search was performed in five databases, namely Scopus, Cochrane Central Register of Controlled Trials (CENTRAL), Web of Science, Embase, and MEDLINE (via PubMed) without any search restrictions from inception to 15th Sept, 2020. The following search key was used: (NASH OR steatohepatitis OR "metabolic associated fatty liver disease" or "Non-alcoholic Fatty Liver Disease" OR "Nonalcoholic Fatty Liver Disease" OR MAFLD OR NAFLD) AND ("COVID 19" OR "Wuhan virus" OR "coronavirus" OR "2019 nCoV" OR "SARS-Cov-2").

After the removal of duplicates with a reference manager software (EndNote X9, Clarivate Analytics, Philadelphia, PA, USA), papers for title, abstract, and full-text were screened by two independent authors separately according to a predetermined set of rules. In the case of any disagreement, a consensus was reached after discussion with a third author.

Eligible studies reported on $(\mathrm{P})$ patients with confirmed SARSCoV-2 infection and compared the outcomes of patients (I and C) with and without MAFLD or NAFLD to each other. The outcomes (O) were severe COVID-19, ICU admission, and inhospital mortality. Studies with cohort or case-control design ( $>5$ participants) were considered eligible. The severity of COVID19 was classified according to the guidelines on the Diagnosis and Treatment of COVID-19 issued by the National Health Commission of China (Supplementary Table 2) (15). When there were multiple publications using data with overlapping study populations, we included the one with greater sample size.

\section{Data Extraction}

Two independent review authors performed data extraction from eligible studies into a standardized data collection form. A third independent author resolved disagreements.

The following information was extracted from each study: first author, year of publication, digital object identifier, study design, study period, the number of centers, study site (country), demographic characteristics of the study population, the number of patients, the number of participants with and without MAFLD or NAFLD separately, the number of patients with event (severe COVID-19, ICU admission, mortality) with and without MAFLD or NAFLD separately, and, if available, odds ratios for COVID-19 severity, ICU admission, and mortality regarding MAFLD or NAFLD, and parameters included in multivariate adjustments.

\section{Statistical Analysis}

All calculations were performed by Stata 15 data analysis and statistical software (Stata Corp LLC, College Station, TX, USA). All outcomes were handled as dichotomous variables, and odds ratios (ORs) with 95\% confidence intervals (95\% CIs) were calculated (reference groups: patients without NAFLD or MAFLD). Random effects model was used to calculate the pooled estimates using the DerSimonian-Laird method (16). A $p$-value of $<0.05$ was considered statistically significant. Forest plots were used to present the results of the meta-analyses.

Heterogeneity was tested with $I^{2}$ and $\chi^{2}$ tests. As suggested by the Cochrane Handbook (17), $I^{2}$ values were interpreted as "might not be important" (0-40\%), "moderate" (30$60 \%)$, "substantial" (50-90\%), and "considerable" (75-100\%) heterogeneity, with a $p<0.1$ considered significant (18).

We were unable to assess the presence of publication bias because of the low number of studies included in each analysis. 


\section{Assessment of Risk of Bias}

Two independent review authors carried out the assessment. Discrepancies were resolved by third-party arbitration. We used the modified version of the Quality in Prognostic Studies (QUIPS) tool (19) as per the recommendations of the Cochrane Prognosis Methods Group (20). Methodological details of the assessment are summarized in Supplementary Appendix 1.

\section{RESULTS}

\section{Search and Selection}

The selection process is detailed in Figure 1. We identified 319 records in five databases for evaluation. After the removal of duplicates and careful selection, 25 articles were eligible for fulltext assessment. Altogether, 10 papers were eligible for qualitative and quantitative synthesis $(9,10,21-27)$, however, we excluded one due to overlapping study population (12).

\section{Characteristics of the studies included}

The main characteristics of the studies are summarized in Table 1. Two articles recruited subjects from the USA, one from Israel, and another six from China. Except for two prospective study, all were retrospective cohort studies. MAFLD was defined in all studies based on the consensus by Eslam et al. (8); NAFLD was defined by the presence of hepatic steatosis on imaging. The proportion of patients with MAFLD and NAFLD ranged from 28 to $50 \%$, and from 6 to $38 \%$, respectively, across studies. Eligibility criteria of the studies included are presented in Supplementary Table 3.

\section{Quantitative Syntheses}

In our meta-analysis, we included a total of six studies with 7,284 patients evaluating the severity of COVID-19, the proportion of severe COVID-19 ranged from 10 to $19 \%$. Three articles with 7,433 patients reported on the need for ICU admission, the proportion of ICU admission ranged from 6 to $38 \%$.

MAFLD was associated with an increased risk of severe COVID-19 compared to the non-MAFLD group [28 vs. $13 \%$, respectively; $\mathrm{OR}=2.61, \mathrm{CI}: 1.75-3.91$ in a homogenous dataset $\left(I^{2}=0.0 \%\right.$ with $\left.\left.p=0.483\right)\right]$ (Figure 2A). Similarly, in the NAFLD vs. non-NAFLD comparison, NAFLD proved to be a risk factor as well [ 36 vs. $12 \%$, respectively; $\mathrm{OR}=5.22, \mathrm{CI}: 1.94-14.03$ in a heterogenous dataset $\left(I^{2}=85.1 \%\right.$ with $\left.p=0.001\right)$ ] (Figure 2B).

Although patients with NAFLD were more likely to be admitted to ICU compared to those without NAFLD, the difference did not reach the level of statistical significance [24 vs. $7 \%$, respectively; $\mathrm{OR}=2.29$, CI: $0.79-6.63$ in a heterogenous dataset $\left(I^{2}=85.1 \%\right.$ with $\left.p=0.001\right)$ ] (Figure 2C).

\section{Qualitative Syntheses}

We were not able to make a meta-analytical analysis for the MAFLD vs. non-MAFLD comparison on the rate of ICU admission, however, two studies $(10,27)$ reported on ICU admission. Gao et al. (27) in non-diabetic MAFLD patients found an increased risk of intensive care requirement in those with critical illness compared to non-MAFLD patients $(p=0.003$, 4.6 vs. $0.0 \%$, respectively). Zhou et al. (10), in a matched cohort of MAFLD and non-MAFLD patients, found a significantly increased risk of the composite outcome of severe and critical COVID-19 in MAFLD patients compared to the non-MAFLD group (OR = 3.65, CI: 1.31-10.16).

Regarding in-hospital mortality, Hashemi et al. (23) found similar rates in COVID-19 patients with NAFLD compared to those without NAFLD ( $p=0.54,16.4$ vs. $13.2 \%$ ).

A summary of multivariate logistic regression analyses from each study included can be found in Supplementary Table 4. Most of the studies adjusted for age, sex, and underlying conditions in multivariate analysis. In the study of Ji et al. (24), NAFLD was associated with COVID-19 progression (adjusted $\mathrm{OR}=6.4$, CI: 1.5-31.2). Bramante et al. (9) found an increased odds of hospital admission in COVID-19 patients with NAFLD (adjusted OR $=2.04, \mathrm{CI}: 1.55-2.69$ ). Based on two studies, ICU admission (adjusted OR $=1.70, \mathrm{CI}$ : 1.20-2.40; adjusted $\mathrm{OR}=2.3$, CI: $1.27-4.17$, respectively) and need for mechanical ventilation (adjusted OR $=1.98, \mathrm{CI}: 1.28-3.06$; adjusted $\mathrm{OR}=2.15, \mathrm{CI}$ : 1.18-3.91, respectively) were also increased with $\operatorname{NAFLD}(9,23)$. Finally, NAFLD was not found to increase in-hospital mortality in COVID-19 (adjusted OR = 0.99, CI: 0.54-1.77) (9).

On the other hand in COVID-19 patients with MAFLD, Mahamid et al. (25) found that MAFLD was associated with severe COVID-19 in both sexes (adjusted OR = 3.29, CI: 3.283.58 for men, adjusted $\mathrm{OR}=3.25, \mathrm{CI}$ : 3.09-3.47 for women), independently of MS. In the study of Zhou et al. (21), an association between the presence of MAFLD and COVID-19 severity was observed in patients younger than 60 years (adjusted $\mathrm{OR}=2.67$, CI: $1.13-6.34$ ), but not in those above 60 years (adjusted OR $=0.61, \mathrm{CI}: 0.18-2.03$ ). In non-diabetic patients, Gao et al. (27) found an increased risk of severe COVID19 only in MAFLD patients with both obesity and metabolic dysregulation (adjusted OR $=5.25$, CI: $1.23-22.33$ ), but the difference was non-significant if only one of the criteria was present $(\mathrm{OR}=2.60, \mathrm{CI}: 0.47-14.42)$.

\section{Risk of Bias Assessment}

Among the included studies, three were of moderate overall risk of bias. All the other studies were rated to carry high overall risk of bias. The summary of risk of bias assessment is shown in Supplementary Figures 1-5.

\section{DISCUSSION}

In our meta-analysis, we aimed to analyse the association between MAFLD or NAFLD and COVID-19 outcomes. Based on our results, we identified that MAFLD is associated with 2.6 times higher risk of severe COVID-19 compared to the nonMAFLD group. In the NAFLD vs. non-NAFLD comparison, we found a five-times increased risk of severe COVID-19. The rate of the ICU admission was higher in NAFLD patients compared to those without NAFLD; however, the difference was statistically non-significant. Finally, we did not find any difference regarding in-hospital mortality in COVID-19 patients with MAFLD or NAFLD in qualitative synthesis.

Previous reviews have assessed the effect of MAFLD or NAFLD in COVID-19 patients, however, to our knowledge, this 


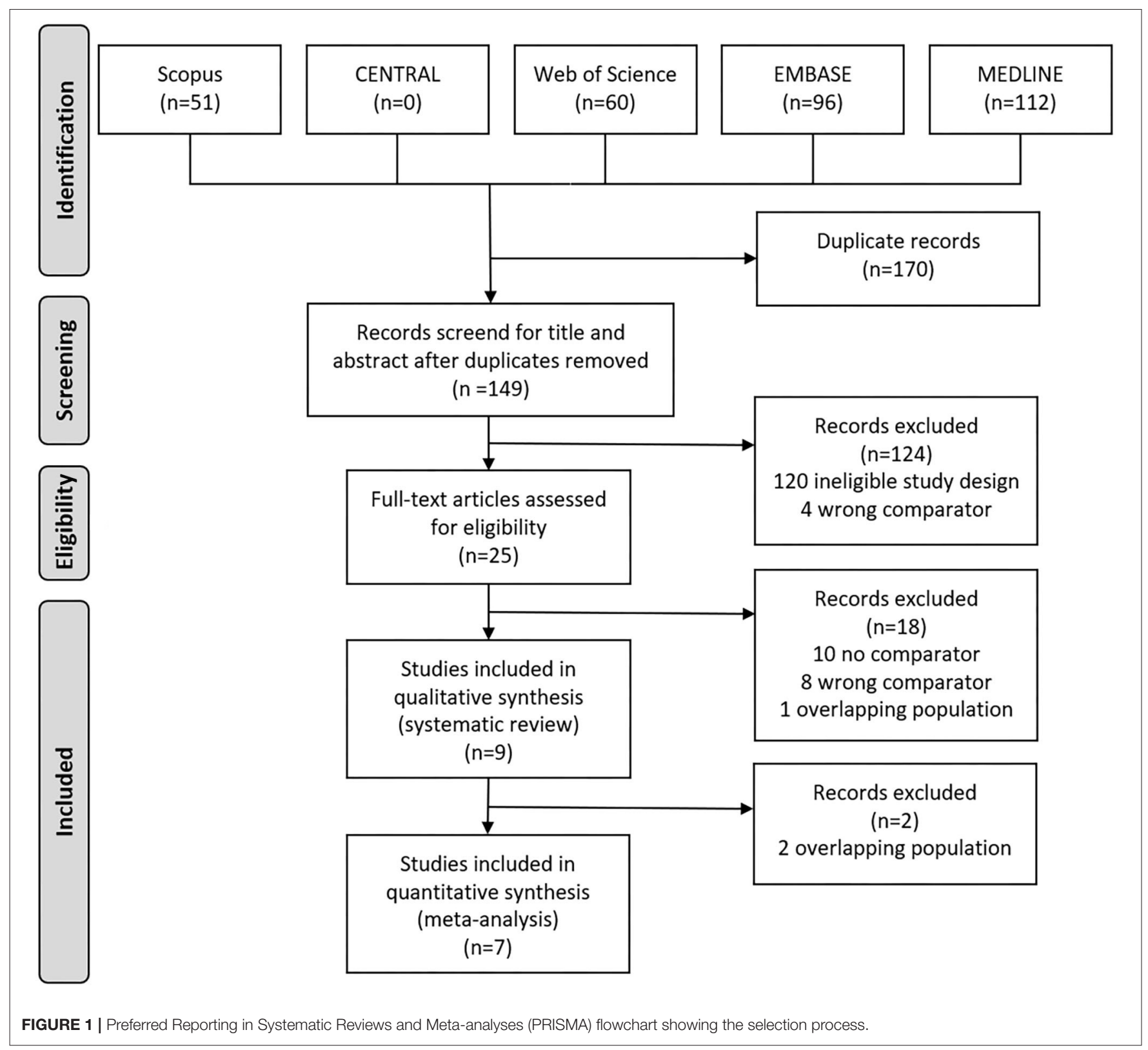

is the first systematic review and meta-analysis in this topic $(6,11,28)$.

Six of the included articles reported on covariate adjusted results $(9,21,23-25,27)$, most of them supporting our conclusion on the impact of MAFLD and NAFLD in COVID-19. We could not perform a meta-analytical evaluation of these results, as there were different outcomes assessed and covariates adjusted for. Based on these results, MAFLD and NAFLD are associated with a higher risk of severe COVID-19 and ICU admission both in uniand multi-variate analyses.

Previously several comorbidities such as hypertension, diabetes, extreme obesity, and cardiovascular disease were reported to be associated with worse prognosis in COVID19 patients $(3,5)$. Several meta-analyses reported on the role of CLD in COVID-19 (29, 30). Based on our previous paper (31), pre-existing liver diseases and on-admission liver-related laboratory results predicted a more severe outcome in SARSCoV-2 infection. However, none of the articles performed subgroup analysis based on the underlying liver condition.

The association between MAFLD or NAFLD and COVID-19 severity is certainly multifactorial. MS and elements of it have been already linked to untoward outcomes in COVID-19 (32). In type 2 diabetes, the second most common comorbidity in COVID-19, the poor prognosis is likely the consequence of the whole clinical picture: poor glucose control, advanced age, and diabetes-associated comorbidities (33). Obesity is associated with chronic inflammation compromising the immune response resulting in an increased risk of more severe infections $(34,35)$, 
TABLE 1 | Basic characteristics of included studies in the systematic review and meta-analysis.

\begin{tabular}{|c|c|c|c|c|c|c|c|}
\hline Author & Country & $\begin{array}{l}\text { Total } \mathbf{N}^{0} \text { of } \\
\text { patients }\end{array}$ & Female \% & Age (year) ${ }^{\dagger}$ & $\begin{array}{c}\mathrm{N}^{0} \text { of patients with MAFLD or } \\
\text { NAFLD (\% of total) }\end{array}$ & \multicolumn{2}{|c|}{ Outcome(s) } \\
\hline \multicolumn{8}{|c|}{ NAFLD vs. no-NAFLD comparison } \\
\hline (9) & USA & 6,802 & 44 & 46 & $373(5.5)$ & $\begin{array}{l}\text { Severe COVID-19/ } \\
\text { ICU admission }\end{array}$ & $930(13.67)$ \\
\hline & & & & & & & $55(15.67)$ \\
\hline \multirow[t]{2}{*}{ (22) } & China & 280 & 48 & 43 & $86(31)$ & $\begin{array}{l}\text { Severe COVID-19/ } \\
\text { ICU admission }\end{array}$ & $28(10)$ \\
\hline & & & & & & & $18(6.43)$ \\
\hline (26) & China & 310 & 52 & 47 & $94(30)$ & Severe COVID-19 & $50(16.13)$ \\
\hline$(21)^{\ddagger}$ & China & 327 & ND & ND & $93(28)$ & Severe COVID-19 & $59(18)$ \\
\hline$(10)^{\ddagger}$ & China & 110 & 26 & 42 & $55(50)$ & ICU admission & $3(2.73)$ \\
\hline
\end{tabular}

${ }^{\dagger}$ mean or median, $\neq$ prospective study.

COVID-19, coronavirus disease 2019; ICU, intensive care unit; NAFLD, non-alcoholic fatty liver disease; ND, not defined; MAFLD, metabolic associated fatty liver disease.

on the other hand, obesity is also a significant risk factor for ICU admission and invasive mechanical ventilation (5). In patients with diabetes, hyperinflammatory response, microvascular endothelial dysfunction, and microthrombi formation may contribute to the poorer outcomes in COVID-19 (6).

Similarly, based on previous reports (26), in patients with MAFLD, a pro-inflammatory state could exacerbate the SARSCoV-2 induced cytokine storm. Ji et al. (24) found in a retrospective study that COVID-19 patients with MAFLD had a poorer prognosis, two-fold higher prevalence of severe disease course, and also higher viral shedding time, and more liver failure during hospitalization.

In the included studies several differences between study populations were highlighted. Increased liver fat content was associated with a higher risk of symptomatic COVID-19 in univariate analysis (OR $=1.85,95 \%$ OR: 1.05-3.25) (36). Moreover, the authors found that obesity and concomitant $>10 \%$ liver fat content exposed an increased risk of severe COVID-19 $(\mathrm{OR}=2.96,95 \% \mathrm{CI}: 1.12-7.78)$; those obese patients with normal liver fat content $(<5 \%)$ showed no elevation of risk $(\mathrm{OR}=0.36$, 95\% CI: 0.1-1.26). The importance of the liver fat content has been pointed out in the study by Bramante et al. (9) as well.

On the other hand, the presence of fibrosis in MAFLD patients is another risk factor for severity of COVID-19, independently of metabolic comorbidities. Based on Targher et al. (12), the severity of COVID-19 significantly increased with the extent of liver fibrosis; those with a FIB-4 score higher than 2.67 had the highest risk of developing severe COVID-19 (OR = 5.73, 95\% CI: 1.84-17.9). After adjustment for sex, obesity, and diabetes, this considerable association persisted (adjusted OR $=2.91,95 \%$ CI: $1.20-7.06$ ).

The same authors demonstrated that the presence of MAFLD together with a neutrophil-to-lymphocyte ratio (NLR) higher than 2.8 is associated with a higher risk of severe COVID-19 compared to patients without MAFLD and with normal NLR (26). NLR was previously highlighted to be a useful, widely available prognostic factor in the early phase of SARS-CoV-2 infection (37).

Another interesting point was reported by Zhou et al. (21). In COVID-19 patients with MAFLD under 60 years, a more than 4-fold risk of severe COVID-19 was observed compared to those without MAFLD (OR = 3.97, 95\% CI: 1.89-8.35); after adjusting for covariates (adjusted OR $=2.67,95 \%$ CI: $1.13-6.34$ ) the risk remained significantly higher. In contrast, in multivariate analysis in elderly patients, MAFLD was not associated with severity of COVID-19. These results need to be supported by further cohort analysis.

None of the studies reported on long-term outcomes in COVID-19.

\section{Strengths and Limitations}

Considering the strengths of our meta-analysis, a rigorous methodology was followed, and we did not deviate from the pre-study protocol, except for including mortality in our investigated outcomes. Several limitations must be considered when interpreting our results. First of all, we could not analyse in-hospital mortality in our meta-analysis. Secondly, our study involved data from only nine articles. It must be noted that, 


\begin{tabular}{|c|c|c|c|c|c|}
\hline Authors & & $\begin{array}{c}\text { OR } \\
(95 \% \mathrm{Cl})\end{array}$ & $\begin{array}{c}\text { Event/ } \\
\text { exposed }\end{array}$ & $\begin{array}{c}\text { Event/ } \\
\text { Non- } \\
\text { exposed }\end{array}$ & $\begin{array}{c}\text { Weight } \\
\%\end{array}$ \\
\hline \multicolumn{6}{|l|}{ (A) Severe COVID-19 in MAFLD vs non-MAFLD } \\
\hline Zhou ZJ et al (2020) & $\longrightarrow$ & $2.16(1.20,3.88)$ & $25 / 93$ & $34 / 234$ & 47.48 \\
\hline Targher G et al (2020) & $\longrightarrow$ & $2.77(1.49,5.14)$ & $25 / 94$ & $25 / 216$ & 42.43 \\
\hline Mahamid M et al (2020) & & $5.03(1.41,17.89)$ & $8 / 22$ & $5 / 49$ & 10.09 \\
\hline Overall $\left(I^{2}=0.0 \%, p=0.483\right)$ & & $2.61(1.75,3.91)$ & $58 / 209$ & $64 / 499$ & 100 \\
\hline \multicolumn{6}{|l|}{ (B) Severe COVID-19 in NAFLD vs non-NAFLD } \\
\hline Huang R et al (2020) & & $1.80(0.81,4.00)$ & $12 / 86$ & $16 / 194$ & 31.96 \\
\hline Bramante CT et al (2020) & 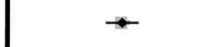 & $4.75(3.81,5.92)$ & $148 / 373$ & $782 / 6429$ & 39.53 \\
\hline Ji D et al (2020) & & $19.59(7.19,53.37)$ & $34 / 76$ & $5 / 126$ & 28.51 \\
\hline Overall $\left(I^{2}=85.1 \%, p=0.001\right)$ & & $5.22(1.94,14.03)$ & $194 / 535$ & $803 / 6749$ & 100 \\
\hline \multicolumn{6}{|l|}{ (C) ICU admission in NAFLD vs non-NAFLD } \\
\hline Huang R et al (2020) & & $0.86(0.30,2.49)$ & $5 / 86$ & $13 / 194$ & 27.79 \\
\hline Hashemi N et al (2020) & $\rightarrow$ & $1.92(1.08,3.40)$ & $29 / 57$ & $103 / 294$ & 34.62 \\
\hline Bramante CT et al (2020) & $\rightarrow$ & $5.53(4.25,7.19)$ & $88 / 373$ & $340 / 6429$ & 37.59 \\
\hline Overall $\left(I^{2}=85.1 \%, p=0.001\right)$ & & $2.29(0.79,6.63)$ & $122 / 516$ & $456 / 6917$ & 100 \\
\hline \multicolumn{6}{|l|}{ NOTE: Weights are from random effects analysis } \\
\hline .01 & 10 & 100 & & & \\
\hline Protective factor & Risk Factor & & & & \\
\hline
\end{tabular}

FIGURE 2 | Odds ratio for COVID-19 severity in patients (A) with MAFLD vs. non-MAFLD, (B) with NAFLD vs. non-NAFLD, and odds ratio for ICU admission in patients (C) with NAFLD vs. non-NAFLD.

we detected significant differences despite the limited study populations, however, with considerable statistical heterogeneity in some of our results. Most of the studies included a low number of patients. The number of studies prevented us from analyzing publication bias ( $<10$ articles). Most of the articles were published from Asian countries; therefore, it is difficult to generalize these results. Also the rate of MAFLD and NAFLD in the study populations differed from the rate reported in the general population. The definition of MAFLD was homogenous, however, NAFLD was diagnosed using different methods across studies. Finally, data came mostly from retrospective studies, with most of them carrying high risk of bias.

\section{CONCLUSION}

\section{Implication for Practice}

In conclusion, the presence of MAFLD or NAFLD is associated with a more severe COVID-19. The presence of further metabolic dysfunction may have additional negative impact on the course of COVID-19. Based on this, health-care providers should follow MAFLD patients cautiously and preventive measures should be taken in these high-risk populations. Therefore, weight loss and regular physical activity should be encouraged in MAFLD patients. 


\section{Implication for Research}

The underlying mechanisms behind our results are still poorly understood. Further research is needed to understand the effect of the pro-inflammatory state associated with MAFLD on the cytokine storm caused by SARS-CoV-2 infection. The severity of COVID-19 should be further stratified based on the severity of MAFLD to explore further high-risk patient groups. Further research is needed to support our results as well as other outcomes, such as mortality, should be analyzed.

\section{DATA AVAILABILITY STATEMENT}

The original contributions generated for the study are included in the article/Supplementary Material, further inquiries can be directed to the corresponding author/s.

\section{AUTHOR CONTRIBUTIONS}

GP and PJH designed the research and the study concept. ZS and SV performed the data extraction. NF analyzed and interpreted the data. FD and $\mathrm{KO}$ performed the quality and risk assessment, $\mathrm{PJH}, \mathrm{BE}, \mathrm{SV}, \mathrm{SK}, \mathrm{PH}$, and GP wrote the article. BE, PH, and GP conducted a critical revision of the manuscript for important intellectual content. All of the co-authors granted final approval of the version of the article to be published.

\section{REFERENCES}

1. Coronavirus disease (COVID-19) Weekly Epidemiological Update and Weekly Operational Update (2020). Available online at: https://www.who.int/ emergencies/diseases/novel-coronavirus-2019/situation-reports

2. Ragab D, Salah Eldin H, Taeimah M, Khattab R, Salem R. The COVID19 cytokine storm; what we know so far. Front Immunol. (2020) 11:1446. doi: 10.3389/fimmu.2020.01446

3. Zádori N, Váncsa S, Farkas N, Hegyi P, Eross B, Szakó L, et al. The negative impact of comorbidities on the disease course of COVID-19. Intensive Care Med. (2020) 46:1784-6. doi: 10.1007/s00134-020-06161-9

4. Garg S, Kim L, Whitaker M, O’Halloran A, Cummings C, Holstein H, et al. Hospitalization rates and characteristics of patients hospitalized with laboratory-confirmed coronavirus disease 2019-COVID-NET, 14 States, March 1-30, 2020. Mortal Wkly Rep. (2020) 69:458-464. doi: 10.15585/mmwr.mm6915e3

5. Földi M, Farkas N, Kiss S, Zádori N, Váncsa S, Szakó L, et al. Obesity is a risk factor for developing critical condition in COVID-19 patients: a systematic review and meta-analysis. Obes Rev. (2020) 21:e13095. doi: 10.1111/obr.13095

6. Dongiovanni P, Meroni M, Longo M, Fracanzani AL. MAFLD in COVID19 patients: an insidious enemy. Expert Rev Gastroenterol Hepatol. (2020) 14:867-72. doi: 10.1080/17474124.2020.1801417

7. Serné EH, de Jongh RT, Eringa EC, IJzerman RG, Stehouwer CD. Microvascular dysfunction. Hypertension. (2007) 50:20411. doi: 10.1161/HYPERTENSIONAHA.107.089680

8. Eslam M, Sanyal AJ, George J. MAFLD: a consensus-driven proposed nomenclature for metabolic associated fatty liver disease. Gastroenterology. (2020) 158:1999-2014.e1. doi: 10.1053/j.gastro.2019.11.312

9. Bramante C, Tignanelli CJ, Dutta N, Jones E, Tamariz L, Clark JM, et al. Nonalcoholic fatty liver disease (NAFLD) and risk of hospitalization for Covid-19. medRxiv [Preprint]. (2020). doi: 10.1101/2020.09.01.20185850

10. Zhou Y-J, Zheng KI, Wang X-B, Sun Q-F, Pan K-H, Wang T-Y, et al. Metabolic-associated fatty liver disease is associated with severity of COVID19. Liver Int. (2020) 40:2160-3. doi: 10.1111/liv.14575

\section{FUNDING}

Study costs are covered by an Economic Development and Innovation Operative Program Grant (GINOP 2.3.2-15-2016-00048) and by a Human Resources Development Operational Program Grant (EFOP-3.6.2-16-2017-00006), both co-financed by the European Union (European Regional Development Fund) within the framework of the Széchenyi 2020 Program. Sponsors had no role in the design, data collection, analysis, interpretation, and preparations of the manuscript.

\section{ACKNOWLEDGMENTS}

The analysis was conducted on behalf of the Translational Action and Research Group against Coronavirus (KETLAK) Study Group. Future study costs will be covered by Economic Development and Innovation Operative Program Grant (GINOP-2.3.4-15-2020-00010).

\section{SUPPLEMENTARY MATERIAL}

The Supplementary Material for this article can be found online at: https://www.frontiersin.org/articles/10.3389/fmed. 2021.626425/full\#supplementary-material

11. Sharma P, Kumar A. Metabolic dysfunction associated fatty liver disease increases risk of severe Covid-19. Diabetes Metab Syndr. (2020) 14:8257. doi: 10.1016/j.dsx.2020.06.013

12. Targher G, Mantovani A, Byrne CD, Wang X-B, Yan H-D, Sun Q-F, et al. Risk of severe illness from COVID-19 in patients with metabolic dysfunctionassociated fatty liver disease and increased fibrosis scores. Gut. (2020) 69:1545. doi: 10.1136/gutjnl-2020-321611

13. Assante G, Williams R, Youngson NA. Is the increased risk for MAFLD patients to develop severe COVID-19 linked to perturbation of the gut-liver axis? J Hepatol. (2020) doi: 10.1016/j.jhep.2020.05.051

14. Moher D, Liberati A, Tetzlaff J, Altman DJB. Preferred reporting items for systematic reviews and meta-analyses: the PRISMA statement. BMJ. (2009) 339:b2535. doi: 10.1136/bmj.b2535

15. Zu ZY, Jiang MD, Xu PP, Chen W, Ni QQ, Lu GM, et al. Coronavirus disease 2019 (COVID-19): a perspective from China. Radiology. (2020) 296:E15E25. doi: 10.1148/radiol.2020200490

16. DerSimonian R, Laird N. Meta-analysis in clinical trials. Controll Clin Trials. (1986) 7:177-88. doi: 10.1016/0197-2456(86)90046-2

17. Higgins JP, Thomas J, Chandler J, Cumpston M, Li T, Page MJ, et al. Cochrane Handbook for Systematic Reviews of Interventions. Oxford, UK: John Wiley \& Sons (2019).

18. Higgins JP, Thompson SG, Deeks JJ, Altman DGJB. Measuring inconsistency in meta-analyses. BMJ. (2003) 327:557-60. doi: 10.1136/bmj.327.74 14.557

19. Hayden JA, van der Windt DA, Cartwright JL, Côté P, Bombardier CJAoim. Assessing bias in studies of prognostic factors. Ann Intern Med. (2013) 158:280-6. doi: 10.7326/0003-4819-158-4-201302190-00009

20. Riley RD, Moons KGM, Snell KIE, Ensor J, Hooft L, Altman DG, et al. A guide to systematic review and meta-analysis of prognostic factor studies. BMJ. (2019) 364:k4597. doi: 10.1136/bmj.k4597

21. Zhou Y-J, Zheng KI, Wang X-B, Yan H-D, Sun Q-F, Pan K-H, et al. Younger patients with MAFLD are at increased risk of severe COVID19 illness: a multicenter preliminary analysis. J Hepatol. (2020) 73:71921. doi: $10.1016 /$ j.jhep.2020.04.027 
22. Huang R, Zhu L, Wang J, Xue L, Liu L, Yan X, et al. Clinical features of patients with COVID-19 with nonalcoholic fatty liver disease. Hepatol Commun. (2020) 64:1758-68. doi: 10.1002/hep4.1592

23. Hashemi N, Viveiros K, Redd WD, Zhou JC, McCarty TR, Bazarbashi AN, et al. Impact of chronic liver disease on outcomes of hospitalized patients with COVID-19: A multicentre United States experience. Liver Int. (2020) 40:2515-21. doi: 10.1111/liv.14583

24. Ji D, Qin E, Xu J, Zhang D, Cheng G, Wang Y, et al. Non-alcoholic fatty liver diseases in patients with COVID-19: retrospective study. J Hepatol. (2020) 73:451-3. doi: 10.1016/j.jhep.2020.03.044

25. Mahamid M, Nseir W, Khoury T, Mahamid B, Nubania A, Sub-Laban K, et al. Nonalcoholic fatty liver disease is associated with COVID-19 severity independently of metabolic syndrome: a retrospective case-control study. Eur J Gastroenterol Hepatol. (2020). doi: 10.1097/MEG.0000000000001902. [Epub ahead of print].

26. Targher G, Mantovani A, Byrne CD, Wang XB, Yan HD, Sun QF, et al. Detrimental effects of metabolic dysfunction-associated fatty liver disease and increased neutrophil-to-lymphocyte ratio on severity of COVID-19. Diabetes Metab Syndr. (2020) 46:505-7. doi: 10.1016/j.diabet.2020.06.001

27. Gao F, Zheng KI, Wang X-B, Yan H-D, Sun Q-F, Pan K-H, et al. Metabolic associated fatty liver disease increases coronavirus disease 2019 disease severity in nondiabetic patients. J Gastroenterol Hepatol. (2020) 36:2047. doi: 10.1111 /jgh.15112

28. Portincasa P, Krawczyk M, Smyk W, Lammert F, Di Ciaula A. COVID-19 and non-alcoholic fatty liver disease: two intersecting pandemics. Eur J Clin Invest. (2020) 50:e13338. doi: 10.1111/eci. 13338

29. Lippi G, de Oliveira MHS, Henry BM. Chronic liver disease is not associated with severity or mortality in Coronavirus disease 2019 (COVID-19): a pooled analysis. Eur J Gastroenterol Hepatol. (2021) 33:114-5. doi: 10.1097/MEG.000000000 0001742

30. Kovalic AJ, Satapathy SK, Thuluvath PJ. Prevalence of chronic liver disease in patients with COVID-19 and their clinical outcomes: a systematic review and meta-analysis. Hepatol Int. (2020) 14:612-20. doi: 10.1007/s12072-020-10078-2

31. Váncsa S, Hegyi PJ, Zádori N, Szakó L, Vörhendi N, Ocskay K, et al. Pre-existing liver diseases and on-admission liver-related laboratory tests in
COVID-19: a prognostic accuracy meta-analysis with systematic review. Front Med. (2020) 7:743. doi: 10.3389/fmed.2020.572115

32. Williamson E, Walker AJ, Bhaskaran KJ, Bacon S, Bates C, Morton CE, et al. OpenSAFELY: factors associated with COVID-19-related hospital death in the linked electronic health records of 17 million adult NHS patients. medRxiv [Preprint]. (2020). doi: 10.1101/2020.05.06.20092999

33. Apicella M, Campopiano MC, Mantuano M, Mazoni L, Coppelli A, Del Prato S. COVID-19 in people with diabetes: understanding the reasons for worse outcomes. Lancet Diabetes Endocrinol. (2020) 8:78292. doi: 10.1016/S2213-8587(20)30238-2

34. Lighter J, Phillips M, Hochman S, Sterling S, Johnson D, Francois F, et al. Obesity in patients younger than 60 years is a risk factor for COVID19 hospital admission. Clin Infect Dis. (2020) 71:896-7. doi: 10.1093/cid/c iaa415

35. Poulain M, Doucet $M$, Major GC, Drapeau V, Sériès F, Boulet L-P, et al. The effect of obesity on chronic respiratory diseases: pathophysiology and therapeutic strategies. Can Med Assoc J. (2006) 174:1293. doi: 10.1503/cmaj.051299

36. Roca-Fernandez A, Dennis A, Nicolls R, McGonigle J, Kelly M, Banerjee R. High liver fat associates with higher risk of developing symptomatic covid-19 infection - initial uk biobank observations. medRxiv [Preprint]. (2020). doi: 10.1101/2020.06.04.20122457

37. Ciccullo A, Borghetti A, Zileri Dal Verme L, Tosoni A, Lombardi F, Garcovich $\mathrm{M}$, et al. Neutrophil-to-lymphocyte ratio and clinical outcome in COVID19: a report from the Italian front line. Int J Antimicrob Agents. (2020) 56:106017. doi: 10.1016/j.ijantimicag.2020.106017

Conflict of Interest: The authors declare that the research was conducted in the absence of any commercial or financial relationships that could be construed as a potential conflict of interest.

Copyright (C 2021 Hegyi, Váncsa, Ocskay, Dembrovszky, Kiss, Farkas, Erőss, Szakács, Hegyi and Pár. This is an open-access article distributed under the terms of the Creative Commons Attribution License (CC BY). The use, distribution or reproduction in other forums is permitted, provided the original author(s) and the copyright owner(s) are credited and that the original publication in this journal is cited, in accordance with accepted academic practice. No use, distribution or reproduction is permitted which does not comply with these terms. 